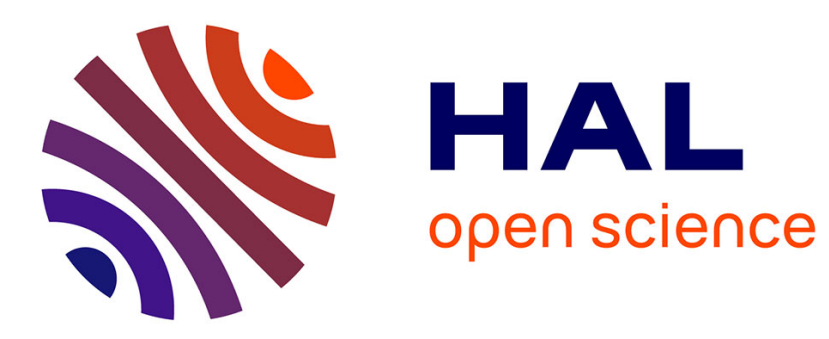

\title{
Broadband MAS NMR spectroscopy in the low-power limit
}

Kevin J Sanders, Andrew Pell, Sebastian Wegner, Clare Grey, Guido Pintacuda

\section{- To cite this version:}

Kevin J Sanders, Andrew Pell, Sebastian Wegner, Clare Grey, Guido Pintacuda. Broadband MAS NMR spectroscopy in the low-power limit. Chemical Physics Letters, 2018, 697, pp.29-37. 10.1016/j.cplett.2018.01.028 . hal-01744691

\section{HAL Id: hal-01744691 \\ https://hal.science/hal-01744691}

Submitted on 2 May 2018

HAL is a multi-disciplinary open access archive for the deposit and dissemination of scientific research documents, whether they are published or not. The documents may come from teaching and research institutions in France or abroad, or from public or private research centers.
L'archive ouverte pluridisciplinaire HAL, est destinée au dépôt et à la diffusion de documents scientifiques de niveau recherche, publiés ou non, émanant des établissements d'enseignement et de recherche français ou étrangers, des laboratoires publics ou privés. 


\title{
Broadband MAS NMR Spectroscopy in the Low-Power Limit
}

\author{
Kevin J. Sanders ${ }^{\mathrm{a}}$, Andrew J. Pell ${ }^{\mathrm{b}}$, Sebastian Wegner $^{\mathrm{c}}$, Clare P. Grey ${ }^{\mathrm{d}}$, Guido Pintacuda ${ }^{\mathrm{a}, *}$ \\ ${ }^{a}$ Institut des Sciences Analytiques (CNRS UMR 5280, ENS de Lyon, UCB Lyon 1), Université de Lyon, 5 rue de la Doua, 69100 \\ Villeurbanne, France \\ ${ }^{b}$ Department of Materials and Environmental Chemistry, Arrhenius Laboratory, Stockholm University, SE-106 91 Stockholm, Sweden \\ ${ }^{c}$ Bruker Biospin GmbH, Silberstreifen, D-76287 Rheinstetten, Germany \\ ${ }^{d}$ University of Cambridge, Chemistry Department, Lensfield Road Cambridge CB2 1EW, United Kingdom
}

\begin{abstract}
We investigate the performance of broadband adiabatic inversion pulses in the high-power (short high-powered adiabatic pulse, SHAP) and low-power (single-sideband-selective adiabatic pulse, $\mathrm{S}^{3} \mathrm{AP}$ ) RF regimes on a spin system subjected to large anisotropic interactions. We show by combined experimental results and spin dynamics simulations that when the magic-angle spinning rate exceeds $100 \mathrm{kHz} \mathrm{S}{ }^{3}$ APs begin outperforming SHAPs. This is especially true for low-gamma nuclei, such as ${ }^{6} \mathrm{Li}$ in paramagnetic Li-ion battery materials. Finally, we show how $\mathrm{S}^{3} \mathrm{APs}$ can be improved by combining multiple waveforms sweeping over multiple sidebands simultaneously, in order to produce inverted sideband profiles free from intensity biasing.
\end{abstract}

Keywords: Adiabatic pulses, ultrafast MAS, paramagnetic NMR, broadband NMR, Li-ion battery

\section{Introduction}

Nuclear magnetic resonance spectroscopy (NMR) is an important technique used for structural characterization in materials science, chemistry, and biology. However, NMR studies suffer from low sensitivity due primarily to the inherently low nuclear spin polarization. This effect is particularly troublesome for nuclei with low gyromagnetic ratio and low natural abundance, such as ${ }^{6} \mathrm{Li},{ }^{13} \mathrm{C},{ }^{15} \mathrm{~N}$, or ${ }^{29} \mathrm{Si}$, as the sensitivity scales with the electromotive force induced in the NMR coil, $\mathcal{E}(t)$, which itself is proportional to $\gamma^{3}$ :

$$
\mathcal{E}(t) \propto \gamma^{3} N_{\mathrm{s}}
$$

where $\gamma$ is the gyromagnetic ratio and $N_{\mathrm{s}}$ is the number of spins in the system. Before taking into account the number of spins present, this means that the sensitivity relative to ${ }^{1} \mathrm{H}$ for ${ }^{7} \mathrm{Li}$ is $\sim 5.9 \%$, and only a miniscule $\sim 0.3 \%$ for ${ }^{6} \mathrm{Li}$ ! Add to this the low natural abundance of ${ }^{6} \mathrm{Li}(7.59 \%)$ and considering that the signal-to-noise ratio grows only by the square root of the number of co-added transients, we see that it is obviously crucial to maximize the strength of the signal in each scan in order to collect a spectrum in a reasonable amount of time for low-abundance, low-gamma nuclei.

Furthermore, in solid-state NMR measurements, poor sensitivity and resolution are often observed due to large

\footnotetext{
*Corresponding author

Email address: guido.pintacuda@ens-lyon.fr (Guido Pintacuda)
}

anisotropic interactions that severely broaden signals $[1$, $2]$. Broadening caused by the shift anisotropy (SA) often is of the order of $10 \mathrm{~s}$ of $\mathrm{kHz}$ for diamagnetic samples and $100 \mathrm{~s}$ of $\mathrm{kHz}$ in the case of paramagnetic samples, while broadening due to quadrupolar interactions can be many $\mathrm{MHz}$ [2]. Magic-angle spinning (MAS) [3, 4] is ubiquitously used to improve sensitivity and resolution in solidstate NMR experiments by turning a broad static powder pattern in to a series of narrower bands separated by the rotational frequency with intensities that are characteristic of the anisotropy experienced by the nucleus. The maximum sensitivity and resolution is achieved using the highest possible MAS rotation rate available, and in the infinite speed limit, all of the signal collapses to a single narrow band centered about the isotropic resonance frequency.

The acquisition of MAS spectra of nuclei exhibiting very broad anisotropic spinning sideband patterns is inherently challenging due primarily to limitations in the the bandwidth of the pulse used and the detection bandwidth of the probe. A spin echo experiment, $\frac{\pi}{2}-\tau_{\text {echo }}-\pi$, must typically be used to acquire a flat baseline, however, conventional RF $\pi$ pulses often have bandwidths limited to around 100 $\mathrm{kHz}$ using typical maximum pulse powers available in MAS probes. It is possible to overcome bandwidth issues by using a shorter refocusing pulse, for instance a $\frac{\pi}{2}$ or even a $\frac{\pi}{4}$ pulse, but this approach results in severe sensitivity losses as $\frac{\pi}{2}$ or $\frac{\pi}{4}$ pulses do not efficiently refocus coherences. Another approach to solve this issue could be the use of frequency stepping $[5,6,7,8]$ to collect multiple spectra at different frequency offsets and sum them to yield a spectrum without truncation due to pulse imperfections 
or limited probe bandwidth. This technique is suitable for highly abundant and sensitive spins such as ${ }^{1} \mathrm{H}$ and ${ }^{31} \mathrm{P}$, however in practice it is time consuming for sensitive studies of low- $\gamma$, low-abundant nuclei.

A more sensitive alternative is to use broadband swept-frequency pulses for refocusing coherences exhibiting broad anisotropic spinning sideband manifolds. Many pulse shapes have been introduced to solve this problem, such as the hyperbolic secant pulse [9], the Wide, UniformRate Smooth-Truncation (WURST) pulse [10], and the tanh/tan pulse [11]. These broadband pulses have many uses in a variety of magnetic resonance applications and in the case of solid-state studies, WURST pulses have been used to great effect in ultra-wideline spectroscopy [12] of static powders $[13,14,15]$, and have also been used in broadband adiabatic inversion cross polarization (BRAIN$\mathrm{CP})$ experiments of static powders [16, 17].

Broadband swept-frequency pulses were not robustly applied to fast MAS $\left(\nu_{\mathrm{R}}>30 \mathrm{kHz}\right)$ studies of samples with very large shift anisotropies until the introduction of short high-powered adiabatic pulses (SHAPs) [18], which typically utilize the tanh/tan pulse shape with very broad frequency sweeps $\left(1 \leq \Delta \nu_{\mathrm{RF}} \leq 20 \mathrm{MHz}\right.$, Figure 1 left panel) and short pulse times $(\leq 50 \mu \mathrm{s})$ and RF field strengths several times the MAS rotation frequency. These pulses have been particularly successful primarily in the application to paramagnetic samples [18, 19, 20, 21, 22, 23, 24, 25] and have been incorporated into conventional MAS NMR experiments such as the spin echo [18], the Carr-PurcellMeiboom-Gill echo train acquisition technique [26], a heteronuclear correlation variant of the transferred-echo double resonance experiment [19], and the magic angle turning experiment [20]. Indeed, these pulses have proven quite useful in broadband MAS NMR measurements, however it must be stressed that SHAPs demand very high RF fields, $\nu_{1}$, falling in the so-called high-power regime, $\nu_{1} \gg \nu_{\mathrm{R}}$, and this relationship between MAS rate and the required $\mathrm{RF}$ field seems to grow at a rate that is more than linear [27]. This is due to the fact that the increased rate of modulation of the anisotropic shift during higher MAS rates necessarily cause SHAPs to require increasing $\mathrm{RF}$ field strengths at increasing MAS rates to maintain the adiabatic condition, and thus keep the magnetization sufficiently locked to the effective field [18]. The use of SHAPs are therefore limited to studies where suitably high RF fields can be achieved [27].

On the other hand, low-power pulses have been long used for a variety of applications, notably selective saturation $[28,29]$. In order to achieve excitation, inversion, or refocusing of broad spinning sideband manifolds with low RF fields, one approach is to use DANTE pulse trains [30]; however, although this method can be used to excite broad spinning sideband manifolds, one must sacrifice bandwidth over the isotropic range of frequencies [27]. Spectra containing multiple isotropic environments are incompletely excited as a result, but it is nevertheless useful to accurately measure SA tensor information for isotropi-
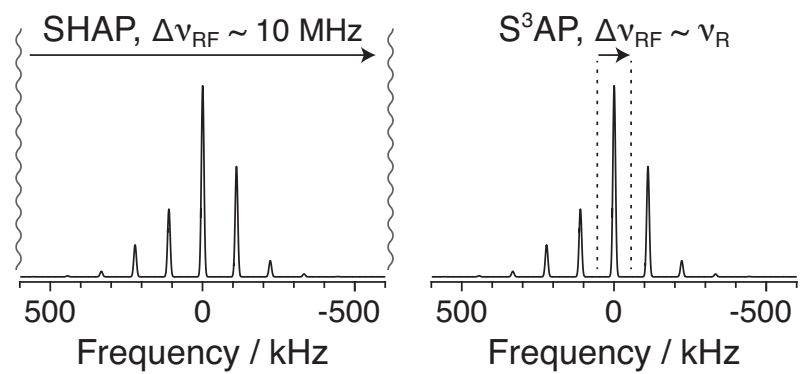

Figure 1: A comparison between the different frequency sweep profiles of a high-power SHAP and a low-power $\mathrm{S}^{3} \mathrm{AP}$ applied to a spin with shift anisotropy parameters $\zeta_{\delta}=250 \mathrm{kHz}$, and $\eta=0.5$ at 111 $\mathrm{kHz}$ MAS.

cally resolved sites [30]. An alternative type of pulse that falls in the low-power regime, $\nu_{1} \ll \nu_{\mathrm{R}}$, and which suffers significantly less than DANTE with respect to limited isotropic bandwidth, was recently proposed for MAS measurements of spectra with broad sideband manifolds and was dubbed the single-sideband-selective adiabatic pulse $\left(\mathrm{S}^{3} \mathrm{AP}\right)[31]$. $\quad \mathrm{S}^{3} \mathrm{APs}$ sweep slowly through only a single sideband (i.e. $\Delta \nu_{\mathrm{RF}} \sim \nu_{\mathrm{R}}$, Figure 1 right panel) and rely on rotary resonance to invert or refocus the entire spinning sideband manifold during a long pulse $\left(\tau_{\mathrm{p}} \gg \tau_{\mathrm{R}}\right)$. This means that over the course of the pulse the instantaneous resonance frequency of the magnetization vector of each crystallite sweeps through the excitation window multiple times, eventually leading to inversion if the adiabatic condition is met. In this way $\mathrm{S}^{3} \mathrm{APs}$ have very low $\mathrm{RF}$ field requirements, and indeed have been shown in silico to demand decreasing RF fields at increasing MAS rates [27]. Using a similar principle, hyperbolic secant pulses and WURST pulses have been used to increase sensitivity for half-integer spin quadrupolar nuclei $[32,33,34]$. S ${ }^{3}$ APs have been used recently in BRAIN-CP measurements under MAS conditions for integer spin [35] and half-integer spin quadrupolar nuclei [36], and were also recently used to achieve efficient population inversion of the ${ }^{14} \mathrm{~N}$ resonances with large quadrupolar couplings in glycine at an MAS rate of $111 \mathrm{kHz}$ [37].

Here we explore the advantages of using high-power tanh/tan SHAPs versus low-power WURST $\mathrm{S}^{3} \mathrm{APs}$ for population inversion in broadband NMR experiments for the case of the paramagnetic lithium ion battery cathode material $\mathrm{LiFe}_{0.25} \mathrm{Mn}_{0.75} \mathrm{PO}_{4}$ [38]. This material exhibits a large SA due to coupling between ${ }^{6,7} \mathrm{Li}$ spins and unpaired electrons in the $\mathrm{Fe}^{\mathrm{II}}$ and $\mathrm{Mn}^{\mathrm{II}}$ metal centers. We use both the ${ }^{6} \mathrm{Li}\left(\gamma_{6} \mathrm{Li}=6.27 \mathrm{MHz} / \mathrm{T}\right)$ and ${ }^{7} \mathrm{Li}\left(\gamma_{7} \mathrm{Li}=\right.$ 16.55 MHz/T) nuclei in order to examine the effect of $\gamma$ on the pulse performance at MAS rates ranging from 40 $\mathrm{kHz}$ to $111 \mathrm{kHz}$. 


\section{Inversion pulses in the high-power and low- power limits}

We start by discussing the use of high-power SHAPs and low-power $\mathrm{S}^{3} \mathrm{APs}$ for population inversion. We note in passing that despite the fact that swept-frequency pulses are most routinely used as refocusing pulses (in magnetic resonance imaging, solution-state NMR, and static solidstate NMR studies in particular), the pulse performance under MAS conditions is much more readily examined in the case of inversion. Therefore the remainder of this manuscript will focus solely on the inversion performance of SHAPs and $\mathrm{S}^{3} \mathrm{APs}$, though we note that $\mathrm{S}^{3} \mathrm{APs}$ may be used for refocusing in the same way that SHAPs have been implemented previously $[18,27,37]$. The theory describing the spin dynamics during SHAPs and $\mathrm{S}^{3}$ APs has been already discussed extensively in prior publications $[18,31,27,37]$ and any further discussion is outside the scope of this work, however we draw two general conclusions from this prior work for the case of $\mathrm{S}^{3} \mathrm{APs}$ : (1) that, in general, the maximum available MAS rate should be used, and (2) that generally the frequency sweep should be swept through the most intense sideband. These conclusions come as a result of the fact that the low-power condition must necessarily be met. Therefore at low-tomoderate MAS rates the available RF field strengths are limited and therefore the low-power condition is often violated, whereas under ultrafast MAS the maximum permitted RF field is higher. Prior results have shown $\mathrm{S}^{3} \mathrm{APs}$ to outperform SHAP pulses under ultrafast $\left(\nu_{\mathrm{R}} \geq 60 \mathrm{kHz}\right)$ MAS rates, but this has been hitherto unconfirmed experimentally.

The performance of SHAPs and $\mathrm{S}^{3} \mathrm{APs}$ under moderate MAS $(40 \mathrm{kHz})$ and ultrafast MAS $(111 \mathrm{kHz})$ are shown in Figure 2 (a) and (b), respectively, which shows the optimal inversion results for each condition and highlights the ability of SHAP pulses to perform comparably better than $\mathrm{S}^{3} \mathrm{AP}$ pulses under moderate MAS conditions. The complete $\mathrm{RF}$ inversion results for ${ }^{6} \mathrm{Li}$ are shown in Figure 2 (c) and (d) for SHAPs and $\mathrm{S}^{3} \mathrm{APs}$ respectively, as a function of RF field strength. They show quite nice agreement between experimental values (filled shapes) and simulated values (solid or dashed lines) for MAS rotation frequencies of 40,62.5, and $111 \mathrm{kHz}$. However, sufficiently high $\mathrm{RF}$ fields for low- $\gamma$ nuclei are extremely difficult to generate. For example, in a $0.7 \mathrm{~mm}$ probe a maximum $\mathrm{RF}$ field strength of $182 \mathrm{kHz}$ was achieved for ${ }^{6} \mathrm{Li}$ with $100 \mathrm{~W}$ input power, and this problem is exacerbated with larger coils. Therefore, the $\mathrm{S}^{3} \mathrm{AP}$ clearly outperforms the SHAP at $111 \mathrm{kHz}$ MAS, shown in Figure 2(b). To the best of our knowledge, this is the first such time that a low power pulse has outperformed a high-power counterpart for broadband inversion of an entire spinning sideband manifold.

The same results were not observed in the study of ${ }^{7} \mathrm{Li}$ NMR at $111 \mathrm{kHz}$ MAS, the results of which are shown in Figure 3, with full simulation results for ${ }^{6} \mathrm{Li}$ and ${ }^{7} \mathrm{Li}$ given in Figure 4. Due to the higher $\gamma$ of ${ }^{7} \mathrm{Li}$, the probe (a)

(b)
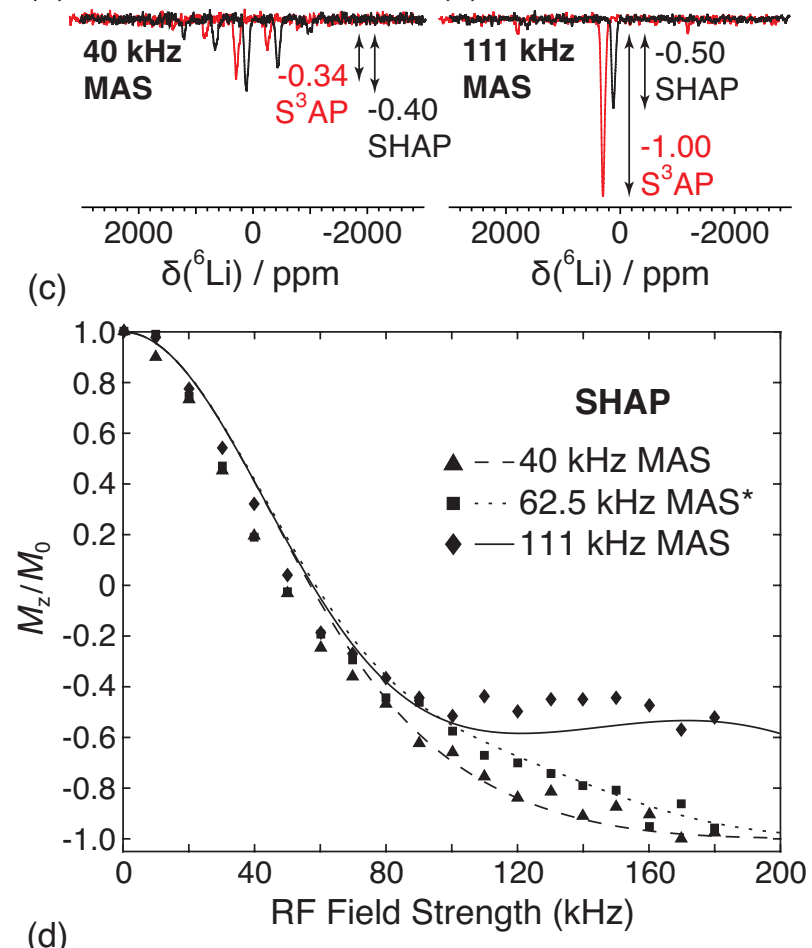

(d)

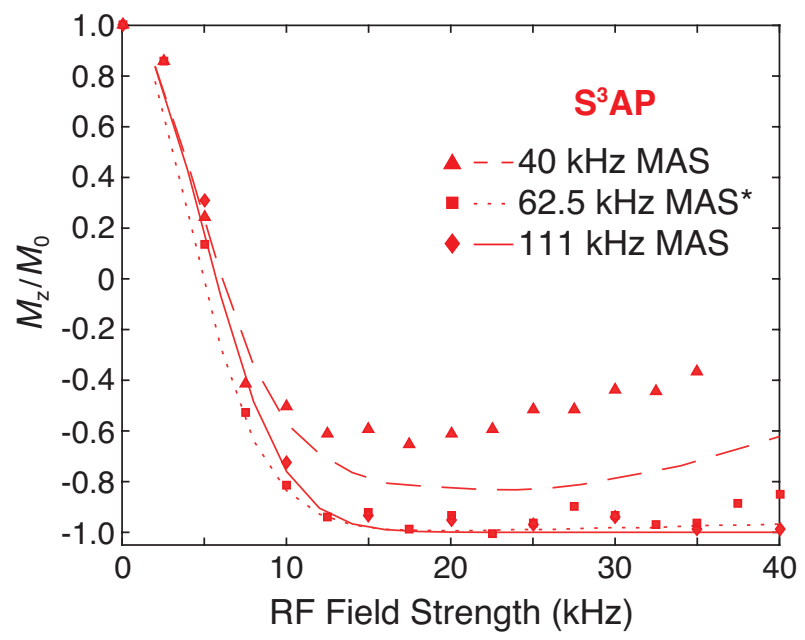

Figure 2: Comparison of inversion efficiencies under an MAS rate of (a) $40 \mathrm{kHz}$, and (b) $111 \mathrm{kHz}$ for a $\operatorname{SHAP}\left(\Delta \nu_{\mathrm{RF}}=10 \mathrm{MHz}\right.$, black $)$ and $\mathrm{S}^{3} \mathrm{AP}\left(\Delta \nu_{\mathrm{RF}}=\nu_{\mathrm{R}}\right.$, red $)$ on the ${ }^{6} \mathrm{Li}$ nucleus, normalized in intensity to the $\mathrm{S}^{3} \mathrm{AP}$-inverted spectrum in (b). The results show the clear difference in performance of SHAPs and $\mathrm{S}^{3} \mathrm{APs}$ depending on the spinning frequency and thus the RF field requirements. Full $\mathrm{RF}$ inversion profiles were determined at 40, 62.5, and $111 \mathrm{kHz}$ MAS for SHAP pulses (c) and $\mathrm{S}^{3} \mathrm{AP}$ pulses (d) as a function of the RF field strength, where the filled shapes represent experimental values and solid or dashed lines are vertical cross sections from the simulations shown in Figure 4 (further simulation details given in the caption). The inverted spectra in (a) and (b) are taken from the optimum points in (c) and (d), corresponding to an RF field strength of 17.5 $\mathrm{kHz}$ for the $\mathrm{S}^{3} \mathrm{AP}$ and $180 \mathrm{kHz}$ for the SHAP in (a), and RF field strenghts of $40.0 \mathrm{kHz}$ for the $\mathrm{S}^{3} \mathrm{AP}$ and $180 \mathrm{kHz}$ for the SHAP in (b). ${ }^{*}$ The simulated inversion profile is an interpolation between simulated inversion profiles with MAS rates of 62 and $63 \mathrm{kHz}$. 
(a)

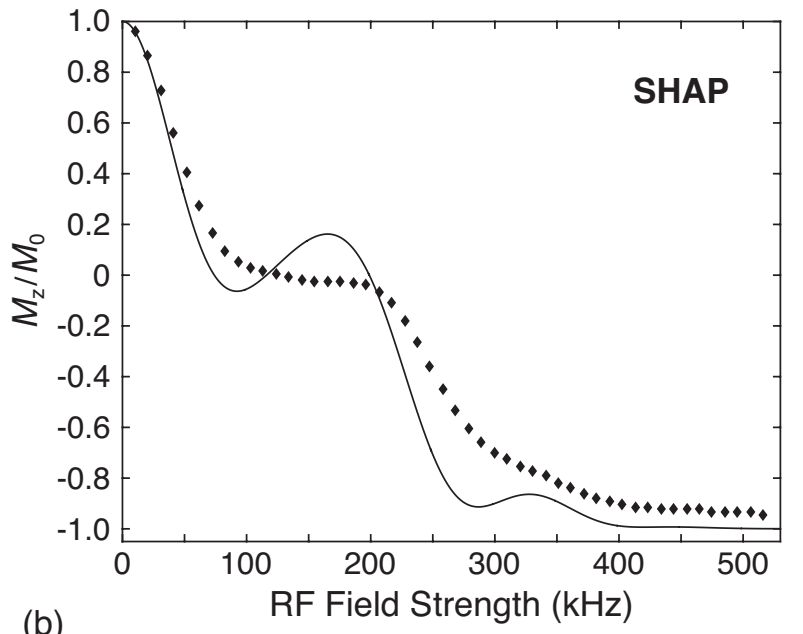

(b)

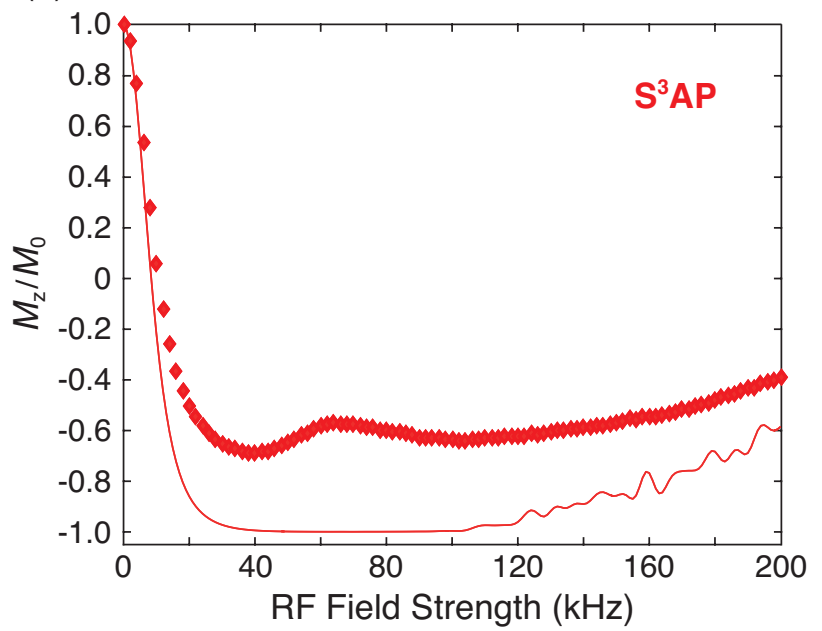

Figure 3: Experimental (diamonds) and simulated (lines) RF inversion profiles for SHAPs $\left(\Delta \nu_{\mathrm{RF}}=10 \mathrm{MHz}\right.$, black $)$ and $\mathrm{S}^{3} \mathrm{APs}$ $\left(\Delta \nu_{\mathrm{RF}}=\nu_{\mathrm{R}}=111 \mathrm{kHz}\right.$, red $)$ on the ${ }^{7} \mathrm{Li}$ nucleus at a MAS rate of $111 \mathrm{kHz}$, with simulated values calculated for a spin with equivalent anisotropic to the fitted experimental values, $\zeta_{\delta}=245 \mathrm{kHz}$ and $\eta=$ 0.65 . was able to provide a sufficiently high RF field of $520 \mathrm{kHz}$, thereby permitting near perfect inversion by SHAP pulses. Surprisingly, the $\mathrm{S}^{3} \mathrm{AP}$ pulse performs significantly worse than was expected from simulation results, and we expect that this results from a combination of deterring effects. First, due to paramagnetic relaxation enhancement from $\mathrm{Mn}^{\mathrm{II}}$ and $\mathrm{Fe}^{\mathrm{II}}$ the ${ }^{7} \mathrm{Li}$ relaxation times are quite short, and as a result the $T_{1}$ of ${ }^{7} \mathrm{Li}$ was measured to be $1.49 \mathrm{~ms}$. As such, relaxation losses during a long inversion pulse may be non-negligible, and assuming pure $T_{1}$ relaxation, would be about $17 \%$ for a $270 \mu \mathrm{s} \mathrm{S}{ }^{3} \mathrm{AP}$, compared to about $1 \%$ for a $18 \mu \mathrm{s}$ SHAP. As the magnetization during the pulse spends a non-negligible time in the transverse plane there will also be additional relaxation losses due to $T_{2}$ relaxation, but these losses are difficult to quantify without extensive modeling and simulations, which are outside the scope of this work. An additional and important effect arises from the inability of the $\mathrm{S}^{3} \mathrm{AP}$ to uniformly excite each crystallite in a microcrystalline powder at low $\mathrm{RF}$ field strengths.

In microcrystalline powders there exist different carousels [39] of crystallites, that is, sets of crystallites with the same Euler angles $\alpha$ and $\beta$ but different values of $\gamma$ (i.e. occupying the same orientation but at different times during sample rotation) that each produce distinct sideband patterns. A feature of $\mathrm{S}^{3} \mathrm{APs}$ is that these pulses inherently perform differently for each carousel, as can be explained by recalling equation 17 from Pell et al. [31], which gives the first-order average Hamiltonian of a swept-frequency pulse in the low-power limit:

$$
\overline{\overline{\tilde{\mathcal{H}}}}=\nu_{1}(t) A_{n} \hat{R}_{z}\left(\phi(t)-\phi_{n}\right) \hat{I}_{x} \hat{R}_{z}\left(\phi(t)-\phi_{n}\right)^{-1}
$$

where $\nu_{1}(t)$ is the time-dependent amplitude of the pulse, $A_{n}$ is the amplitude of the $\mathrm{n}^{t h}$-order sideband through which the pulse sweeps, $\hat{R}_{z}(\theta)$ is a rotation operator representing a spin rotation by an angle $\theta$ about the $z$-axis, $\phi(t)$ is the the time-dependent phase of the pulse, and $\phi_{n}$ is the phase of the sideband through which the pulse sweeps. We see immediately from this that the effective magnetic field of the pulse is scaled by the sideband intensity, $A_{n}$, through which the pulse is swept. This has the important effect for samples with anisotropies much larger than the MAS rate that the pulse will not be effective for any carousel of crystallites whose MAS pattern has small or zero intensity in the band over which the pulse is swept. In this case, the optimal RF field for the powder will not be large enough for a carousel with small $A_{n}$, resulting in a biasing of the intensities of the resulting sideband pattern by incompletely inverting or omitting some carousels of crystallites. This effect can be appreciated in Figure 5(a), which shows the $\mathrm{S}^{3} \mathrm{AP}$-inverted spectrum of ${ }^{7} \mathrm{Li}$ at $111 \mathrm{kHz}$ MAS, where the pulse was swept over the centerband. The residuals show a clear bias due to exclusion of carousels with little intensity in the centerband. A 

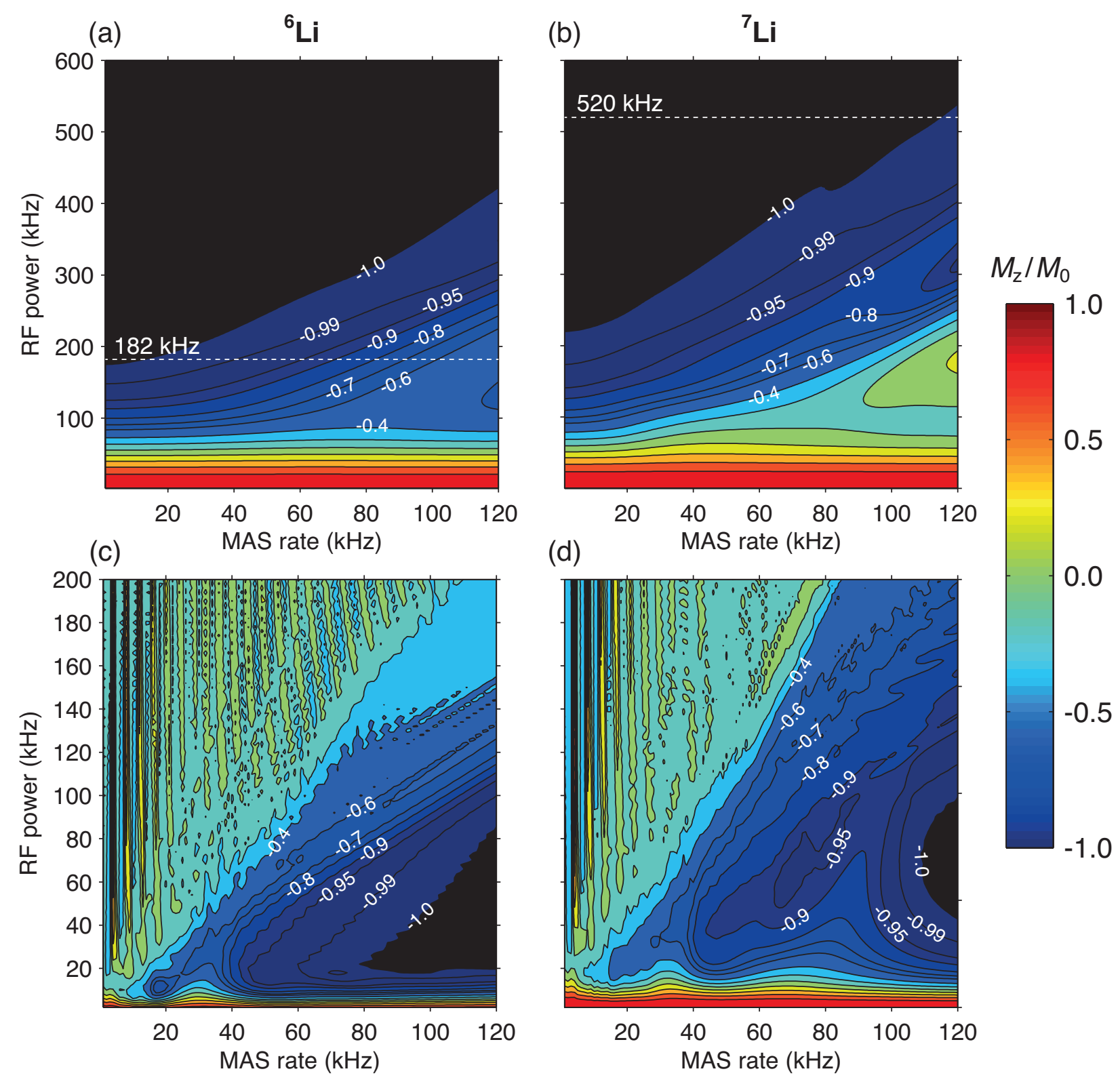

Figure 4: Simulated inversion profile contour plots for a $\operatorname{SHAP}\left(\Delta \nu_{\mathrm{RF}}=10 \mathrm{MHz}\right)\left((\mathrm{a})\right.$ and (b)) and for a $\mathrm{S}^{3} \mathrm{AP}\left(\Delta \nu_{\mathrm{RF}}=\nu_{\mathrm{R}}\right)((\mathrm{c})$ and $(\mathrm{d}))$ as a function of MAS rate and RF field strength, where the white dashed lines denote the maximum available RF field strength for each $\mathrm{Li}$ isotope. The experimental CSA values for ${ }^{6} \mathrm{Li}$ and ${ }^{7} \mathrm{Li}$ were the same and correspond to the fitted experimental values from the ${ }^{7} \mathrm{Li}$ spectrum: $\zeta_{\delta}=245 \mathrm{kHz}$ and $\eta=0.65$ with $\delta=0$ for convenience. It is clear that as the MAS rate and CSA become larger, the RF field requirements for SHAP pulses increase, and in the case of ${ }^{6} \mathrm{Li}$, to an unobtainable practical limit, while the $\mathrm{S}^{3} \mathrm{AP}$ pulse can achieve perfect inversion at $\mathrm{RF}$ field strengths well below the MAS rotation rate in both cases. 


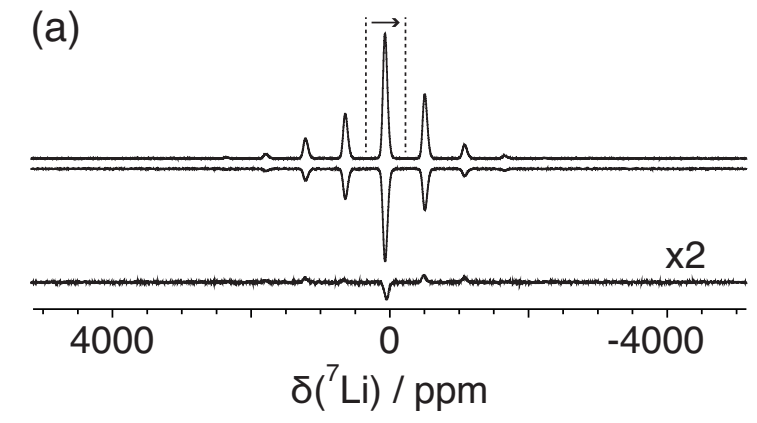

(b)

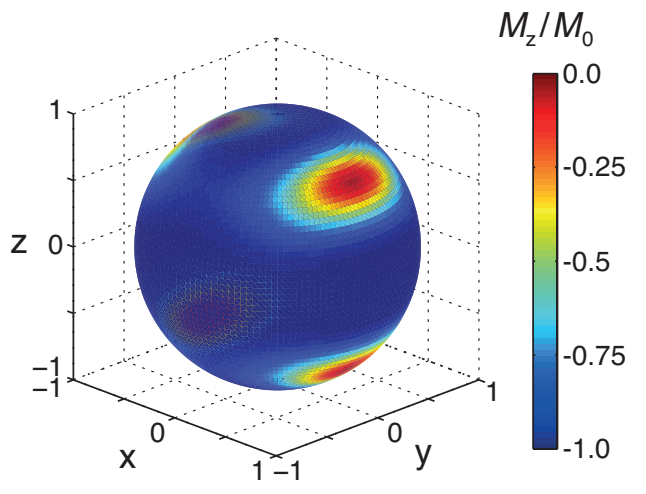

Figure 5: (a) ${ }^{7} \mathrm{Li}$ MAS control spectrum (above), and inverted spectrum (below) using a $\mathrm{S}^{3} \mathrm{AP}$ pulse $\left(\Delta \nu_{\mathrm{RF}}=\nu_{\mathrm{R}}=111 \mathrm{kHz}\right)$ applied to the centerband. The scaled residuals (black, bottom) show a clear biasing of the centerband as a result of the effects discussed in the text. (b) Simulated orientation-dependent inversion profile of a $\mathrm{S}^{3} \mathrm{AP}$ pulse with $\nu_{1, \max }=20 \mathrm{kHz}$ as a function of the crystallite orientation. Each point on the surface of the sphere corresponds to a unique set of Euler angles $(\alpha, \beta)$ and the axis labels $(x, y, z)$ are the Cartesian coordinates for the crystallite orientations after transformation from spherical polar frame to the Cartesian frame. The color of each point corresponds to the size of the $\hat{I}_{z}$ magnetization after the inversion pulse. The results show a clear omission of certain crystallites with small intensity of the centerband, leading to the biasing observed in the residuals in (a). simulation of the orientation-dependent inversion profiles of a $\mathrm{S}^{3} \mathrm{AP}$ pulse with $\nu_{1, \max }=20 \mathrm{kHz}$ was completed by calculating the inversion efficiency as a function of the Euler angles $(\alpha, \beta)$ of a uniformly-sampled set of crystallites, the results of which are shown in Figure 5(b). The results clearly show that certain sets of crystallites with low intensity in the centerband are poorly inverted at low RF field strengths, leading to the biasing observed in Figure $5(\mathrm{a})$.

\section{Simultaneous irradiation over multiple side- bands}

A possible solution to this problem could be to sweep over multiple sidebands simultaneously. The concept of simultaneous irradiation is itself not new [40] but has not yet been applied to spin inversion using broadband sweeps. We begin by recalling the form of a constant amplitude, constant phase pulse:

$$
\overrightarrow{\boldsymbol{\nu}}_{1}=\nu_{1}\left[\cos \left(\phi_{p}\right)_{\mathbf{x}}+\sin \left(\phi_{p}\right)_{\mathbf{y}}\right]
$$

where $\nu_{1}$ is the RF field strength and $\phi_{p}$ is the phase of the RF pulse. This equation can be readily adapted for time-dependent amplitudes and phases:

$$
\overrightarrow{\boldsymbol{\nu}}_{1}(t)=\nu_{1}(t)\left[\cos \left(\phi_{p}(t)\right)_{\mathbf{x}}+\sin \left(\phi_{p}(t)\right)_{\mathbf{y}}\right],
$$

From here, the equation can be modified to permit simultaneous irradiation over two bands. To build a pulse sweeping simultaneously across two bands we will assume that each of the two constituent pulses have the same amplitudes and phase profiles. As such, this new pulse will be described mathematically as the sum of two pulses at with phase offsets $\mathrm{O}_{1}$ and $\mathrm{O}_{2}$ :

$$
\begin{aligned}
\overrightarrow{\boldsymbol{\nu}}_{1}(t)=\nu_{1}(t) & {\left[\left[\frac{1}{2} \cos \left(\phi_{p}(t)+O_{1} t\right)+\frac{1}{2} \cos \left(\phi_{p}(t)+O_{2} t\right)\right]_{\mathbf{x}}\right.} \\
+ & {\left.\left[\frac{1}{2} \sin \left(\phi_{p}(t)+O_{1} t\right)+\frac{1}{2} \sin \left(\phi_{p}(t)+O_{2} t\right)\right]_{\mathbf{y}}\right] . }
\end{aligned}
$$

This can then be expanded using trigonometric identities:

$$
\begin{aligned}
\overrightarrow{\boldsymbol{\nu}}_{1}(t)=\frac{1}{2} \nu_{1}(t)\left[\left[\cos \left(\phi_{p}(t)\right) \cos \left(O_{1} t\right)-\sin \left(\phi_{p}(t)\right) \sin \left(O_{1} t\right)\right.\right. \\
\left.\left.+\cos \left(\phi_{p}(t)\right) \cos \left(O_{2} t\right)-\sin \left(\phi_{p}(t)\right) \sin \left(O_{2} t\right)\right)\right]_{\mathbf{x}} \\
+\left[\sin \left(\phi_{p}(t)\right) \cos \left(O_{1} t\right)+\cos \left(\phi_{p}(t)\right) \sin \left(O_{1} t\right)\right. \\
\left.\left.+\sin \left(\phi_{p}(t)\right) \cos \left(O_{2} t\right)+\cos \left(\phi_{p}(t)\right) \sin \left(O_{2} t\right)\right]_{\mathbf{y}}\right] .
\end{aligned}
$$


If we assume that the pulse is symmetric about zero such at $O_{2}=-O_{1}$, this can be re-written as

$$
\begin{aligned}
\overrightarrow{\boldsymbol{\nu}}_{1}(t)=\frac{1}{2} \nu_{1}(t) & {\left[\left[2 \cos \left(\phi_{p}(t)\right) \cos \left(O_{1} t\right)\right]_{\mathbf{x}}\right.} \\
+ & {\left.\left[2 \sin \left(\phi_{p}(t)\right) \cos \left(O_{1} t\right)\right]_{\mathbf{y}}\right] }
\end{aligned}
$$

and now the term $\cos \left(O_{1} t\right)$ can be factored, yielding the general equation

$$
\overrightarrow{\boldsymbol{\nu}}_{1}(t)=\nu_{1}(t) \cos \left(O_{1} t\right)\left[\cos \left(\phi_{p}(t)\right)_{\mathbf{x}}+\sin \left(\phi_{p}(t)\right)_{\mathbf{y}}\right]
$$

so we see that this is an equation of the same form as a normal RF pulse but with the term $\cos \left(O_{1} t\right)$ superimposed on the amplitude. This results in a pulse that sweeps simultaneously over two bands with equal RF field strength. It is worth stressing that this result produces simultaneous sweeps in the same direction, as compared to "diverging" or "converging" sweeps (in opposite directions) [41] produced by a simple cosine modulation.

For measurements where the desired pulse spacing is a multiple of the rotor frequency, $\nu_{\mathrm{R}}$, this formalism can be rewritten as

$$
\overrightarrow{\boldsymbol{\nu}}_{1}(t)=\nu_{1}(t) \cos \left(\frac{2 \pi \Delta n \nu_{\mathrm{R}} t}{2}\right)\left[\cos \left(\phi_{p}(t)\right)_{\mathbf{x}}+\sin \left(\phi_{p}(t)\right)_{\mathbf{y}}\right]
$$

where $\Delta n$ corresponds to the separation of the irradiated bands in terms of sideband order, noting that the transmitter should be always placed directly between the two desired sidebands. In practice when the amplitude becomes negative, $180^{\circ}$ is added to the phase of the pulse.

\section{Pulsing on three or more bands}

Now that we have worked through the case of two simultaneous sweeps, it is trivial to set up cases with three or more simultaneous frequency sweeps. In the case of three simultaneous sweeps across three adjacent sidebands we can assume that the offsets $O_{1}, O_{2}$, and $O_{3}$ are set such that $O_{1}=-O_{3}=2 \pi \nu_{\mathrm{R}}$ and $O_{2}=0$. Therefore we can construct the amplitude modulation by considering the pulse to be composed of one normal $\mathrm{S}^{3} \mathrm{AP}$ pulse centered about zero, and a pulse simultaneously sweeping over two bands also centered at zero with a band separation $\Delta n=2$. As such the pulse has the form:

$\overrightarrow{\boldsymbol{\nu}}_{1}(t)=\frac{1}{3} \nu_{1}(t)\left[1+2 \cos \left(2 \pi \nu_{\mathrm{R}} t\right)\right]\left[\cos \left(\phi_{p}(t)\right)_{\mathbf{x}}+\sin \left(\phi_{p}(t)\right)_{\mathbf{y}}\right]$ which results in a simultaneous frequency sweep across three adjacent sidebands. This logic could be extended further to sweep simultaneously across four or more bands but this extension is not detailed here.

In order to be able to describe any multiple-band-swept pulse precisely and concisely, we introduce a general notation to describe each pulse: $\mathbf{P} N_{\Delta \nu_{\mathrm{RF}}}^{\Delta n}$, where $\mathbf{P}$ simply denotes that an RF pulse is used, $N$ denotes the number of bands over which the pulse sweeps, $\Delta n$ is the spacing of the bands in terms of sideband order (i.e. $\Delta n=1$ would denote a pulse with adjacent irradiated sidebands), and $\Delta \nu_{\mathrm{RF}}$ is the RF sweep width of each band. With this notation, it is thus necessary to only specify the type of swept-frequency pulse used and the transmitter offset. It should be noted that for $\mathbf{P} N_{\Delta \nu_{\mathrm{RF}}}^{\Delta n}$ pulses with $N>1$, the effective RF field strength applied to each band is the nominal RF field scaled by $N$. For simplicity we report only the nominal RF field (i.e. the frequency measured in a nutation experiment) in all subsequent figures.

The optimal experimental inversion performance of all tested $\mathbf{P} N_{\Delta \nu_{\mathrm{RF}}}^{\Delta n}$ pulses is shown in Figure 6 (a-d) with the optimal sweeps indicated by arrows and the optimized $\mathrm{RF}$ field strengths and inversion efficiency given in text with each panel. Each pulse was optimized experimentally as a function of both transmitter offset and RF field strength with the optimal conditions giving the maximum integrated area. The full results of this optimization are provided in Table 1 of the SI. Contrary to what may be expected, the $\mathbf{P} 1_{\nu_{\mathrm{R}}}\left(\mathrm{S}^{3} \mathrm{AP}\right)$ results shown in Figure 6 (a) provided an optimum inversion when sweeping over the +2 order sideband. This may be explained by considering that more crystallites could have non-negligible intensity, albeit small, in the +2 order sideband thereby permitting more crystallites to be inverted. This observation would also explain the relatively high optimal RF field strength when sweeping over the +2 order sideband, as higher amplitude $\mathrm{RF}$ irradiation must necessarily be applied to compensate for small sideband intensity as previously discussed. A similar result is observed in Figure $6(\mathrm{~b})$, where a $\mathbf{P} 2_{\nu_{\mathrm{R}}}^{1}$ pulse was optimized to sweep over the +1 and +2 order sidebands, again resulting in a very high optimal RF field. In both these cases we observe residuals free of any significant biasing. Sweeping over the 0 and +2 order sidebands with a $\mathbf{P} 2_{\nu_{\mathrm{R}}}^{2}$ pulse unsurprisingly resulted in lower optimal $\mathrm{RF}$ field requirements, shown in Figure 6 (c), due to the high intensity of the centerband, however we observe biasing in the residuals, which could have come as a result of incomplete inversion of crystallites being inverted by pulsing over the +2 band, as the optimized RF field is lower. Finally, sweeping over the $-1,0$, and +1 order sidebands with a $\mathbf{P} 3_{\nu_{\mathrm{R}}}^{1}$ pulse (Figure $6(\mathrm{~d})$ ) resulted in similar inversion efficiency to the $\mathbf{P} 1_{\nu_{\mathrm{R}}}$ pulse but at lower RF field and with small biasing in the residuals, representing a possible compromise between RF field strength and sideband intensity biasing.

In an effort to bolster the conclusions about sideband 
biasing, orientation-dependent inversion profiles of each $\mathbf{P} N_{\Delta \nu_{\mathrm{RF}}}^{\Delta n}$ pulse were completed by calculating the inversion efficiency as a function of the Euler angles $(\alpha, \beta)$ for the experimentally-optimized RF field strength and transmitter offset. The results of these simulations are shown in Figure $6(\mathrm{e}-\mathrm{h})$. In the case of the $\mathbf{P} 1_{\nu_{\mathrm{R}}}\left(\mathrm{S}^{3} \mathrm{AP}\right)$ pulse, the results in Figure 6 (e) show that the inversion is largely uniform, with the exception of a small set of crystallites that have zero intensity in the +2 order sideband. For these crystallites almost no inversion was observed, with an inversion efficiency of $M_{z} / M_{0}=0.93$ in the worst case, resulting in the small biasing observed in the residuals of Figure 6 (a). This effect decreases as the number of sweeps is increased (Figure 6 (f) and (g)), with the best results being given in Figure $6(\mathrm{~h})$ for the $\mathbf{P} 3_{\nu_{\mathrm{R}}}^{1}$ pulse, showing that sweeping over three adjacent sidebands simultaneously yields nearly perfectly uniform inversion. In this case, the worst inversion efficiency was observed for crystallites with low intensity in the centerband, yielding an inversion efficiency of $M_{z} / M_{0}=-0.87$ in the worst case. For all pulses the crystallites with the highest degree of inversion yielded inversion efficiencies of $M_{z} / M_{0}=-1$. In all cases the simulated results do not account for relaxation, and as such small differences may be observed between the simulated and experimental results.

The simulated inversion results for all $\mathbf{P} N_{\Delta \nu_{\mathrm{RF}}}^{\Delta n}$ pulses on a simulated powder is given in Figure 7 as a function of RF field strength and transmitter offset, with powder averaging achieved by simulating $109(\alpha, \beta)$ pairs according to the Lebedev octant scheme [42]. The optimum values for RF field strength and offset were chosen on the basis of maximum integrated area after the inversion pulse, and are denoted with dashed white lines in the first column of Figure 7, with the corresponding cross sections given in the second and third column of Figure 7 for the RF field strength and offset, respectively. The optimized values for RF field strength and offset differ slightly from the experimentally-optimized values in the case of the $\mathbf{P} 1_{\nu_{\mathrm{R}}}$ and $\mathbf{P} 2_{\nu_{\mathrm{R}}}^{1}$ pulses, and this may be explained due to the fact that the simulation does not account for relaxation during the pulse. Although only the optimal condition is denoted in Figure 7, we note that there exist many good combinations of RF field strength and transmitter offset for each pulse, which are given in the deep blue regions of panels $(a, d, g, j)$ of Figure 7 . As such, we observe that in the case of the $\mathbf{P} 1_{\nu_{\mathrm{R}}}$ pulse, efficient inversion can be achieved when sweeping across the +2 order sideband using an $\mathrm{RF}$ field strength of about $100 \mathrm{kHz}$; likewise, efficient inversion can be achieved using the $\mathbf{P} 2_{\nu_{\mathrm{R}}}^{1}$ pulse when sweeping over the +1 and +2 order sidebands with an RF field strength of about $120 \mathrm{kHz}$. Both of these results closely match the experimentally-optimized values as given in Figure 6.

\section{Conclusions}

We have examined the benefits and limitations of both high-power SHAPs and low-power $\mathrm{S}^{3} \mathrm{APs}$ and their appli-
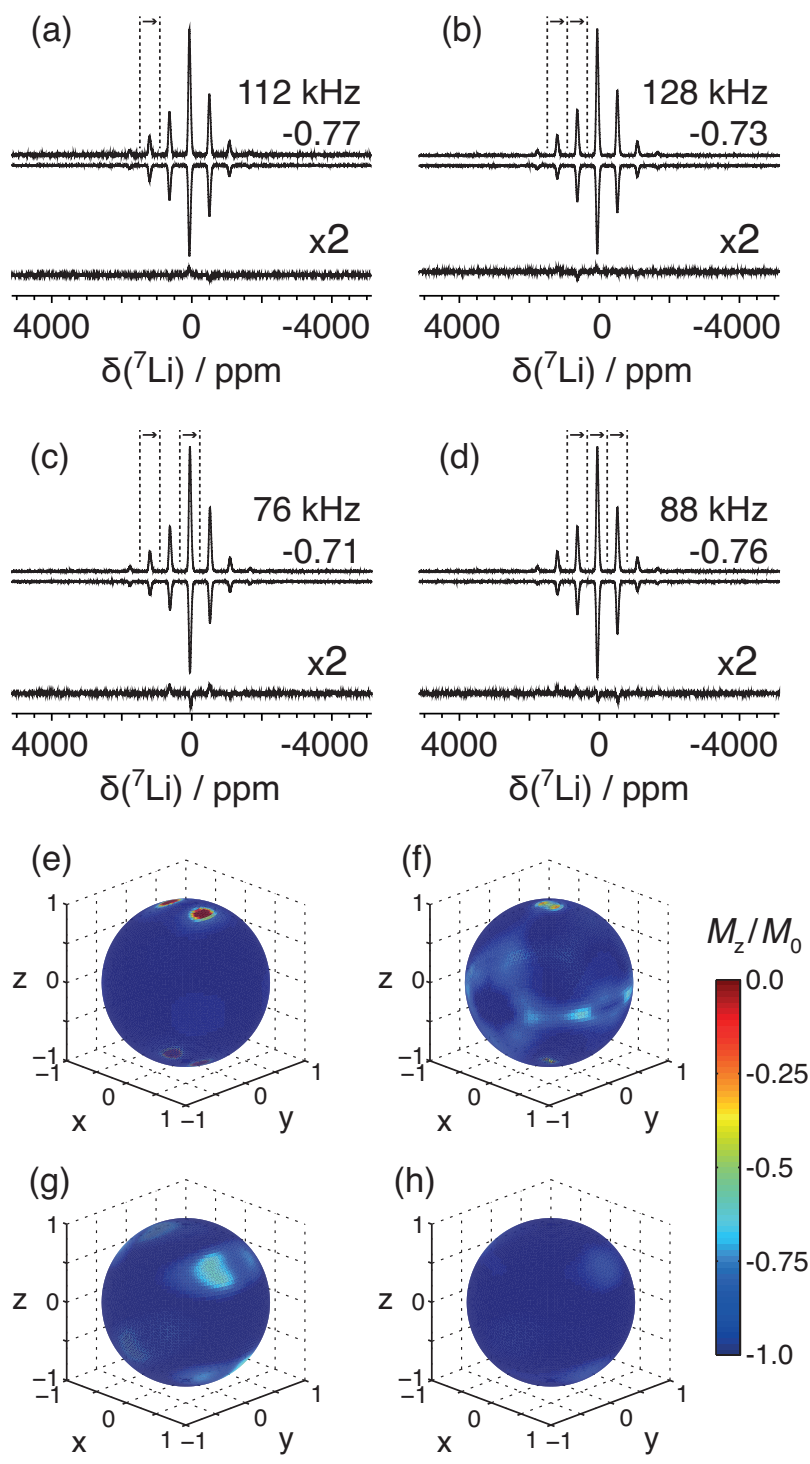

Figure 6: Optimized experimental inversion efficiency and optimized $\mathrm{RF}$ field strength for (a) $\mathbf{P} 1_{\nu_{\mathrm{R}}}\left(\mathrm{S}^{3} \mathrm{AP}\right)$, (b) $\mathbf{P} 2_{\nu_{\mathrm{R}}}^{1}$, (c) $\mathbf{P} 2_{\nu_{\mathrm{R}}}^{2}$, and (d) $\mathbf{P} 3_{\nu_{R}}^{1}$ pulses. The upper spectrum in each panel corresponds to the shifted second echo control spectrum, while the experimental inversion spectrum is below. The residuals for each case are given at the bottom in each panel. The arrows indicate the sidebands over which each experimentally-optimized pulse was swept. Shown below the experimental spectra are simulated orientation-dependent inversion profiles of (e) $\mathbf{P} 1_{\nu_{\mathrm{R}}}\left(\mathrm{S}^{3} \mathrm{AP}\right)$, (f) $\mathbf{P} 2_{\nu_{\mathrm{R}}}^{1}$, (g) $\mathbf{P} 2_{\nu_{\mathrm{R}}}^{2}$, and (h) $\mathbf{P} 3_{\nu_{\mathrm{R}}}^{1}$ pulses using the experimentally-optimized RF field strength and offset in each case. Each point on the surface of the sphere corresponds to a unique set of Euler angles $(\alpha, \beta)$ and the axis labels $(x, y, z)$ are the Cartesian coordinates for the crystallite orientations after transformation from spherical frame to the Cartesian frame. The color of each point corresponds to the size of the $\hat{I}_{z}$ magnetization after the inversion pulse and follow the coloring scheme shown at the right of the simulated data. The results in (e) show that even in the optimized case for $\mathbf{P} 1_{\nu_{\mathrm{R}}}$ pulses, there are sets of crystallite orientations that are excluded from the resulting inverted spectra as a result of these crystallites having spinning sideband patterns with no intensity in the +2 order sideband. On the other hand, the results in (h) demonstrate that sweeping simultaneously over three adjacent sidebands produces much more uniform inversion and therefore a more accurate sideband pattern of the powder after inversion. 

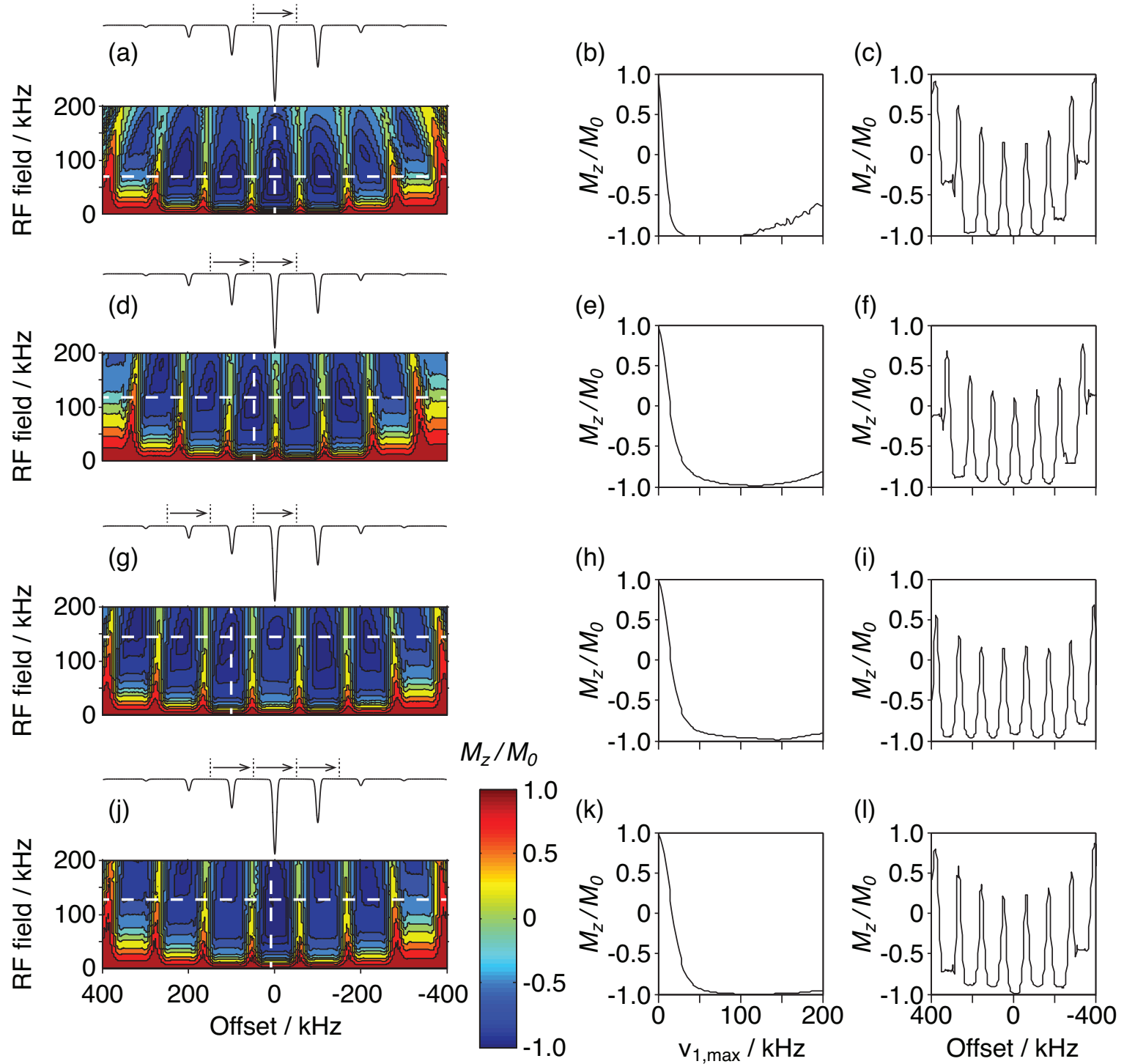

Figure 7: Simulated inversion profiles of a spin with CSA parameters $\zeta_{\delta}=245 \mathrm{kHz}, \eta=0.65$, of a $\mathbf{P} 1_{\nu_{\mathrm{R}}}\left(\mathrm{S}^{3} \mathrm{AP}\right) \mathrm{pulse}(\mathrm{a}-\mathrm{c})$, $\mathbf{P} 2_{\nu_{\mathrm{R}}}^{1}$ pulse (d-f), $\mathbf{P} 2_{\nu_{\mathrm{R}}}^{2}$ pulse (g-i), and a $\mathbf{P} 3_{\nu_{\mathrm{R}}}^{1}$ pulse (j-i) as a function of offset and RF field strength. The optimal offset and RF conditions are denoted by dashed white lines in $(\mathrm{a}, \mathrm{d}, \mathrm{g}, \mathrm{j})$ with the corresponding frequency sweeps shown above each panel, with corresponding vertical (b,e,h,k) and horizontal (c,f,i,l) cross sections provided. 
cation to broadband MAS studies of a sample with large shift anisotropy. Using a MAS rotation rate of 111.111 $\mathrm{kHz}$, we found that in the case of ${ }^{6} \mathrm{Li}$, where the anisotropy is large but $\gamma$ is low, the high-power SHAP performs poorly due primarily to sufficiently large RF fields being unavailable, whereas the low-power $\mathrm{S}^{3} \mathrm{AP}$ provided the maximum achieved sensitivity of all ${ }^{6} \mathrm{Li}$ experiments owing to the fact that only $40 \mathrm{kHz}$ of $\mathrm{RF}$ field strength was required for $100 \%$ inversion. This is the first time that a low-power pulse has been observed to outperform a high-power alternative for an inversion of a broad spinning sideband manifold. This result was not observed for inversion of the high- $\gamma{ }^{7} \mathrm{Li}$ nucleus where sufficiently high $\mathrm{RF}$ fields were available to make SHAPs efficient, while $\mathrm{S}^{3}$ APs suffered sensitivity losses due to relaxation during the pulse and non-uniform inversion of crystallites within the powder.

As a potential way to decrease or even eliminate biasing of sideband intensities caused by $\mathrm{S}^{3} \mathrm{APs}$, we have examined a class of $\mathrm{S}^{3} \mathrm{AP}$-like pulses which sweep simultaneously over multiple bands and can fall comfortably in the low-power regime, and have introduced a notation to describe them. We have found that contrary to what may be expected, $\mathrm{S}^{3} \mathrm{APs}$ could indeed invert ${ }^{7} \mathrm{Li}$ spectra well, but when sweeping not over the centerband. This result comes at the cost of comparatively high $\mathrm{RF}$ field requirements, thereby violating the low-power approximation. We can achieve a similar result - while still falling within the lowpower limit - by pulsing over three adjacent sidebands simultaneously. This represents the possibility of easily implementing low power pulses without extensive experimental optimization, permitting accurate determination of shift anisotropy parameters when efficient high-power pulses are not available due to hardware limitations. We anticipate that this approach to invert spins will be of particular importance for the study of Li-containing battery materials, and more generally, for the study of paramagnetic systems containing low-gamma nuclei.

\section{Experimental}

${ }^{6} \mathrm{Li}$ and ${ }^{7} \mathrm{Li}$ MAS experiments were carried out on the olivine structure lithium-ion battery cathode material $\mathrm{LiFe}_{0.25} \mathrm{Mn}_{0.75} \mathrm{PO}_{4}$ using a Bruker Avance III NMR spectrometer operating at an external field strength of 11.74 T corresponding to Larmor frequences of $73.603 \mathrm{MHz}$ and 194.391 $\mathrm{MHz}$ for ${ }^{6} \mathrm{Li}$ and ${ }^{7} \mathrm{Li}$, respectively. The sample was packed in a $0.7 \mathrm{~mm}$ rotor and spun at the magic angle, $54.736^{\circ}$ relative to the external magnetic field. The magic angle was calibrated by measuring the STMAS [43, 44] spectrum of $\mathrm{Na}_{2} \mathrm{SO}_{4}$ and adjusting the angle until an isotropic line was achieved in the indirect dimension, and was confirmed to have negligible drift $\left(\approx \pm 0.001^{\circ}\right)$ over time and after sample ejection/re-insertion. In all cases the sample temperature was approximately $320 \mathrm{~K}$. A recycle delay of $100 \mathrm{~ms}$ was used for all ${ }^{6} \mathrm{Li}$ experiments while 10 ms was used for ${ }^{7} \mathrm{Li}$ experiments. Further experimental details are given in each figure caption. Due to the hyperfine (a)

(b)
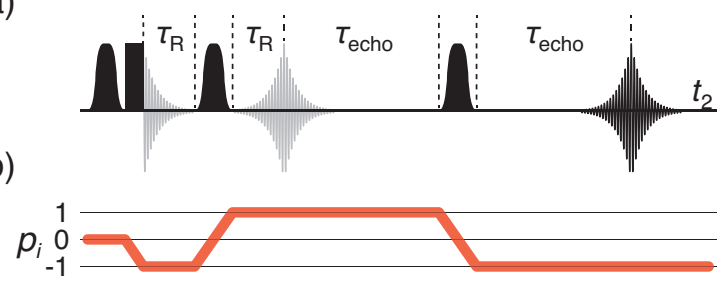

Figure 8: The inverted shifted second echo (a) experiment used in this study. The coherence transfer pathway is given in (b) which utilized a conventional 64-step phase cycle. The black rectanglular pulse represents $\frac{\pi}{2}$ excitation pulses whereas the shaped pulses represent frequency-swept pulses. It is assumed in this sequence that refocusing pulses are rotor-synchronized and likewise that $\tau_{\text {echo }}=N \tau_{\mathrm{R}}$, where $N$ is set to a sufficiently large value to allow acquisition of the entire echo, thereby giving a perfectly flat baseline without truncation artifacts. For ${ }^{6} \mathrm{Li}$ experiments, $N$ was set to 13,20 , and 36 for $40 \mathrm{kHz}, 62.5 \mathrm{kHz}$, and $111.111 \mathrm{kHz}$ MAS experiments, respectively, corresponding to echo shift times of $325 \mu \mathrm{s}, 320 \mu \mathrm{s}$, and $324 \mu \mathrm{s}$. For ${ }^{7} \mathrm{Li}$ measurements at $111.111 \mathrm{kHz} \mathrm{MAS}, N=22$, corresponding to an echo shift time of $198 \mu \mathrm{s}$.

interaction between Li nuclei and the unpaired electrons in the material, Li nuclei experience a large SA. The ${ }^{7} \mathrm{Li} \mathrm{MAS}$ spectrum was fitted using a single anisotropic lineshape, resulting in an experimental chemical shift anisotropy [45], $\zeta_{\delta}=1260 \mathrm{ppm}$, and an asymmetry parameter, $\eta=0.65$. In terms of frequency, this results in anisotropies of $\zeta_{\delta}=$ 92.7 and $245 \mathrm{kHz}$ for ${ }^{6} \mathrm{Li}$ and ${ }^{7} \mathrm{Li}$, respectively.

All swept-frequency pulses used in experiments were constructed using the shape tool in Bruker TopSpin 3.5. Each followed the amplitude, phase, and frequency sweep profiles shown in Table 2 in the SI. All $S^{3} A P s$ were WURST-20 [10] waveforms made of 1000 digitized points with length $\tau_{\mathrm{p}}=270 \mu \mathrm{s}$, and a frequency sweep width, $\Delta \nu$ $=\nu_{\mathrm{R}}$, while all SHAPs were tanh/tan [11] waveforms made of 250 digitized points, with $\xi=10$ and $\tan (\kappa)=20, \Delta \nu=$ $10 \mathrm{MHz}$, and $\tau_{\mathrm{p}}=18 \mu \mathrm{s}$. $\mathbf{P} N_{\Delta \nu_{\mathrm{RF}}}^{\Delta n}$ pulses were constructed simply by adding multiple waveforms with suitable offsets, and otherwise had identical parameters as the $\mathrm{S}^{3} \mathrm{APs}$.

The pulses were examined for inversion performance (i) experimentally using the pulse sequence in Figure 8 (a), and (ii) by spin dynamics simulations using the solid-state simulation program SIMPSON [46] and digitized input pulses constructed by a home-written script in MATLAB. When testing for inversion performance, only the power of the first pulse in Figure 8 (a) was varied, always starting from $0 \mathrm{kHz}$, and the resulting integrals were normalized to the integral of the first point. Spin dynamics simulations were carried out at an external field strength corresponding to a ${ }^{1} \mathrm{H}$ Larmor frequency of $500.196 \mathrm{MHz}$ with powder averaging achieved by simulating $109(\alpha, \beta)$ pairs according to the Lebedev octant scheme [42]. The pulses were applied to a single ${ }^{6} \mathrm{Li}$ or ${ }^{7} \mathrm{Li}$ nucleus using chemical shift anisotropy parameters corresponding to the fitted experimental parameters with $\delta_{\text {iso }}=0$ for convenience. More specific simulation details are provided in the figure captions. 


\section{Acknowledgements}

This work was financially supported by the People Program of the European Union's FP7 (FP7-PEOPLE-2012ITN no. 317127 "pNMR"), the European Research Council (ERC) under the European Union's Horizon 2020 research and innovation program (grant no. 648974 "PMEM-NMR"), and the Agence Nationale de la Recherche (grant no. ANR-15-CE29-0025-01). KJS would like to thank Prof. Philip J. Grandinetti for fruitful discussions about adiabatic pulses.

\section{References}

[1] M. Mehring, Principles of High Resolution NMR in Solids, 2nd Edition, Springer-Verlag, 1983.

[2] M. J. Duer, Introduction to Solid-State NMR Spectroscopy, Blackwell, Oxford, 2004.

[3] E. R. Andrew, A. Bradbury, R. G. Eades, Nuclear Magnetic Resonance Spectra from a Crystal rotated at High Speed, Nature 182 (4650) (1958) 1659-1659, 10.1038/1821659a0.

[4] I. J. Lowe, Free Induction Decays of Rotating Solids, Phys. Rev. Lett. 2 (7) (1959) 285-287.

[5] M. Sananes, A. Tuel, J. Volta, A Study by ${ }^{31}$ P NMR Spin-Echo Mapping of VPO Catalysts, J. Catal. 145 (2) (1994) $251-255$. doi:http://dx.doi.org/10.1006/jcat.1994.1032.

[6] R. W. Schurko, S. Wi, L. Frydman, Dynamic Effects on the Powder Line Shapes of Half-Integer Quadrupolar Nuclei: A Solid-State NMR Study of $\mathrm{XO}^{4-}$ Groups, J. Phys. Chem. A 106 (1) (2002) 51-62. doi:10.1021/jp0130214.

[7] J. T. Ash, P. J. Grandinetti, Solid-state NMR characterization of ${ }^{69} \mathrm{Ga}$ and ${ }^{71} \mathrm{Ga}$ in crystalline solids, Magn. Reson. Chem. 44 (9) (2006) 823-831. doi:10.1002/mrc.1841.

[8] A. J. Pell, R. J. Clément, C. P. Grey, L. Emsley, G. Pintacuda, Frequency-stepped acquisition in nuclear magnetic resonance spectroscopy under magic angle spinning, J. Chem. Phys. 138 (11). doi:http://dx.doi.org/10.1063/1.4795001.

[9] J. Baum, R. Tycko, A. Pines, Broadband and adiabatic inversion of a two-level system by phase-modulated pulses, Phys. Rev. A 32 (1985) 3435-3447. doi:10.1103/PhysRevA.32.3435.

[10] E. Kupce, R. Freeman, Adiabatic pulses for wideband inversion and broadband decoupling, J. Magn. Reson. A 115 (2) (1995) 273 - 276. doi:http://dx.doi.org/10.1006/jmra.1995.1179.

[11] T.-L. Hwang, P. C. van Zijl, M. Garwood, Fast broadband inversion by adiabatic pulses, J. Magn. Reson. 133 (1) (1998) 200 - 203. doi:http://dx.doi.org/10.1006/jmre.1998.1441.

[12] R. W. Schurko, Ultra-wideline solid-state NMR spectroscopy, Accounts Chem. Res. 46 (9) (2013) 1985-1995, pMID: 23745683. arXiv:http://dx.doi.org/10.1021/ar400045t, doi:10.1021/ar400045t.

[13] F. H. Larsen, H. J. Jakobsen, P. D. Ellis, N. C. Nielsen, Sensitivity-enhanced quadrupolar-echo NMR of half-integer quadrupolar nuclei. magnitudes and relative orientation of chemical shielding and quadrupolar coupling tensors, J. Phys. Chem. A 101 (46) (1997) 8597-8606. doi:10.1021/jp971547b

[14] L. A. ODell, R. W. Schurko, QCPMG using adiabatic pulses for faster acquisition of ultra-wideline NMR spectra, Chem. Phys. Lett. 464 (13) (2008) 97 - 102. doi:http://dx.doi.org/10.1016/j.cplett.2008.08.095.

[15] L. A. ODell, A. J. Rossini, R. W. Schurko, Acquisition of ultrawideline NMR spectra from quadrupolar nuclei by frequency stepped WURST-QCPMG, Chem. Phys. Lett. 468 (46) (2009) 330 - 335. doi:http://dx.doi.org/10.1016/j.cplett.2008.12.044.

[16] K. J. Harris, A. Lupulescu, B. E. Lucier, L. Frydman, R. W. Schurko, Broadband adiabatic inversion pulses for cross polarization in wideline solid-state NMR spectroscopy, J. Magn. Reson. 224 (2012) 38 - 47. doi:https://doi.org/10.1016/j.jmr.2012.08.015.

17] K. J. Harris, S. L. Veinberg, C. R. Mireault, A. Lupulescu, L. Frydman, R. W. Schurko, Rapid acquisition of 14 n solid-state nmr spectra with broadband cross polarization, Chem. Eur. J. 19 (48) (2013) 16469-16475. doi:10.1002/chem.201301862.

[18] G. Kervern, G. Pintacuda, L. Emsley, Fast adiabatic pulses for solid-state NMR of paramagnetic systems, Chem. Phys. Lett. 435 (13) (2007) 157 - 162. doi:http://dx.doi.org/10.1016/j.cplett.2006.12.056.

[19] G. Kervern, A. D’Aléo, L. Toupet, O. Maury, L. Emsley, G. Pintacuda, Crystal-Structure Determination of Powdered Paramagnetic Lanthanide Complexes by Proton NMR Spectroscopy, Angew. Chem. Int. Edit. 48 (17) (2009) 3082-3086. doi:10.1002/anie.200805302.

[20] R. J. Clément, A. J. Pell, D. S. Middlemiss, F. C. Strobridge, J. K. Miller, M. S. Whittingham, L. Emsley, C. P. Grey, G. Pintacuda, Spin-transfer pathways in paramagnetic lithium transition-metal phosphates from combined broadband isotropic solid-state mas NMR spectroscopy and DFT calculations, J. Amer. Chem. Soc. 134 (41) (2012) 17178-17185, pMID: 23004936. arXiv:http://dx.doi.org/10.1021/ja306876u, doi:10.1021/ja306876u.

[21] M. Bini, S. Ferrari, C. Ferrara, M. C. Mozzati, D. Capsoni, A. J. Pell, G. Pintacuda, P. Canton, P. Mustarelli, Polymorphism and magnetic properties of $\mathrm{Li}_{2} \mathrm{MSio}_{4}(\mathrm{M}=\mathrm{Fe}, \mathrm{Mn})$ cathode materials, Sci. Rep-UK 3 (2013) 3452 EP -, article.

[22] J. Xu, D. H. Lee, R. J. Clément, X. Yu, M. Leskes, A. J. Pell, G. Pintacuda, X.-Q. Yang, C. P. Grey, $\mathrm{Y}$. S. Meng, Identifying the Critical Role of $\mathrm{Li}$ Substitution in $\mathrm{P} 2-\mathrm{Na}_{x}\left[\mathrm{Li}_{y} \mathrm{Ni}_{z} \mathrm{Mn}_{1-y-z}\right] \mathrm{O}_{2}(0<\mathrm{x}, \quad \mathrm{y}$, $\mathrm{z}<1)$ Intercalation Cathode Materials for HighEnergy Na-Ion Batteries, Chem. Mater. 26 (2) (2014) 1260-1269. arXiv:http://dx.doi.org/10.1021/cm403855t, doi: $10.1021 / \mathrm{cm} 403855 \mathrm{t}$.

[23] F. C. Strobridge, D. S. Middlemiss, A. J. Pell, M. Leskes, R. J. Clement, F. Pourpoint, Z. Lu, J. V. Hanna, G. Pintacuda, L. Emsley, A. Samoson, C. P. Grey, Characterising local environments in high energy density Li-ion battery cathodes: a combined NMR and first principles study of $\mathrm{LiFe}_{x} \mathrm{Co}_{1-x} \mathrm{PO}_{4}$, J. Mater. Chem. A 2 (2014) 11948-11957. doi:10.1039/C4TA00934G.

[24] B. Burcher, K. J. Sanders, L. Benda, G. Pintacuda, E. Jeanneau, A. A. Danopoulos, P. Braunstein, H. Olivier-Bourbigou, P.-A. R. Breuil, Straightforward Access to Stable, 16Valence-Electron Phosphine-Stabilized $\mathrm{Fe}^{0}$ Olefin Complexes and Their Reactivity, Organometallics 36 (3) (2017) 605613. arXiv:http://dx.doi.org/10.1021/acs.organomet.6b00803, doi:10.1021/acs.organomet.6b00803.

[25] A. Bertarello, T. Schubeis, C. Fuccio, E. Ravera, M. Fragai, G. Parigi, L. Emsley, G. Pintacuda, C. Luchinat, Paramagnetic Properties of a Crystalline Iron-Sulfur Protein by Magic-Angle Spinning NMR spectroscopy, Inorg. Chem. 56 (11) (2017) 6624-6629, pMID: 28537393. arXiv:http://dx.doi.org/10.1021/acs.inorgchem.7b00674, doi:10.1021/acs.inorgchem.7b00674.

[26] G. Kervern, S. Steuernagel, F. Engelke, G. Pintacuda, L. Emsley, Absence of Curie Relaxation in Paramagnetic Solids Yields Long ${ }^{1} \mathrm{H}$ Coherence Lifetimes, J. Amer. Chem. Soc. 129 (46) (2007) 14118-14119, pMID: 17960908. arXiv:http://dx.doi.org/10.1021/ja075319z, doi:10.1021/ja075319z.

[27] A. J. Pell, G. Pintacuda, Broadband solid-state MAS NMR of paramagnetic systems, Prog. Nucl. Mag. Res. Sp. 8485 (2015) 33 - 72. doi:http://dx.doi.org/10.1016/j.pnmrs.2014.12.002.

[28] P. Caravatti, G. Bodenhausen, R. Ernst, Selective pulse experiments in high-resolution solid state nmr, Journal of Magnetic Resonance (1969) 55 (1) (1983) 88 - 103. doi:https://doi.org/10.1016/0022-2364(83)90279-2.

[29] M. Shen, J. Trébosc, O. Lafon, Z. Gan, F. Pourpoint, B. Hu, Q. Chen, J.-P. Amoureux, Solid-state nmr indirect detection of 
nuclei experiencing large anisotropic interactions using spinning sideband-selective pulses, Solid State Nuclear Magnetic Resonance 72 (Supplement C) (2015) $104-117$, honor Issue for Jake Schaefer. doi:https://doi.org/10.1016/j.ssnmr.2015.09.003.

[30] D. Carnevale, V. Vitzthum, O. Lafon, J. Tr/'ebosc, J.-P Amoureux, G. Bodenhausen, Broadband excitation in solidstate nmr of paramagnetic samples using delays alternating with nutation for tailored excitation (para-dante), Chemical Physics Letters 553 (Supplement C) (2012) 68 - 76. doi:https://doi.org/10.1016/j.cplett.2012.09.056.

[31] A. J. Pell, G. Kervern, L. Emsley, M. Deschamps, D. Massiot, P. J. Grandinetti, G. Pintacuda, Broadband inversion for MAS NMR with single-sideband-selective adiabatic pulses, J. Chem. Phys. 134 (2). doi:http://dx.doi.org/10.1063/1.3521491.

[32] R. Siegel, T. T. Nakashima, R. E. Wasylishen, Sensitivity enhancement of NMR spectra of half-integer spin quadrupolar nuclei in solids using hyperbolic secant pulses, J. Magn. Reson. 184 (1) (2007) 85 - 100. doi:http://dx.doi.org/10.1016/j.jmr.2006.09.007.

[33] T. T. Nakashima, R. E. Wasylishen, R. Siegel, K. J. Ooms, Sensitivity enhancement of solid-state NMR spectra of half-integer spin quadrupolar nuclei: Double- or singlefrequency sweeps? Insights from the hyperbolic secant experiment, Chem. Phys. Lett. 450 (4) (2008) 417 - 421. doi:http://dx.doi.org/10.1016/j.cplett.2007.11.032.

[34] K. K. Dey, S. Prasad, J. T. Ash, M. Deschamps, P. J. Grandinetti, Spectral editing in solid-state MAS NMR of quadrupolar nuclei using selective satellite inversion, J. Magn. Reson. 185 (2) (2007) 326 - 330. doi:http://dx.doi.org/10.1016/j.jmr.2006.12.013.

[35] S. Wi, R. Schurko, L. Frydman, ${ }^{1} \mathrm{~h}-{ }^{2} \mathrm{~h}$ crosspolarization NMR in fast spinning solids by adiabatic sweeps, J. Chem. Phys. $146 \quad$ (10) (2017) 104201. arXiv:http://dx.doi.org/10.1063/1.4976980, doi:10.1063/1.4976980

[36] S. Wi, C. Kim, R. Schurko, L. Frydman, Adiabatic sweep cross-polarization magic-angle-spinning NMR of half-integer quadrupolar spins, J. Magn. Reson. 277 (2017) 131 - 142. doi:https://doi.org/10.1016/j.jmr.2017.02.021.

[37] A. J. Pell, K. J. Sanders, S. Wegner, G. Pintacuda, C. P. Grey, Low-power broadband solid-state MAS NMR of ${ }^{14}$ N, J. Chem. Phys. 146 (19) (2017) 194202. arXiv:http://dx.doi.org/10.1063/1.4983220, doi:10.1063/1.4983220.

[38] A. Padhi, K. Nanjundaswamy, J. Goodenough, Phosphoolivines as positive-electrode materials for rechargeable lithium batteries, J. Electrochem. Soc. 144 (4) (1997) 1188-1194.

[39] M. H. Levitt, Why do spinning sidebands have the same phase?, Journal of Magnetic Resonance (1969) 82 (2) (1989) 427 - 433. doi:https://doi.org/10.1016/0022-2364(89)90050-4.

[40] A. Kentgens, R. Verhagen, Advantages of double frequency sweeps in static, MAS and MQMAS NMR of spin $\mathrm{i}=3 / 2$ nuclei, Chem. Phys. Lett. 300 (34) (1999) 435 - 443 doi:http://dx.doi.org/10.1016/S0009-2614(98)01402-X.

[41] H. Schäfer, D. Iuga, R. Verhagen, A. P. M. Kentgens, Population and coherence transfer in half-integer quadrupolar spin systems induced by simultaneous rapid passages of the satellite transitions: A static and spinning single crystal nuclear magnetic resonance study, The Journal of Chemical Physics 114 (7) (2001) 3073-3091. arXiv:https://doi.org/10.1063/1.1340576, doi:10.1063/1.1340576.

[42] M. Edén, M. H. Levitt, Computation of orientational averages in solid-state NMR by gaussian spherical quadrature, J. Magn. Reson. 132 (2) (1998) $220 \quad-239$. doi:http://dx.doi.org/10.1006/jmre.1998.1427.

[43] Z. Gan, Isotropic NMR Spectra of Half-Integer Quadrupolar Nuclei Using Satellite Transitions and MagicAngle Spinning, J. Amer. Chem. Soc. 122 (13) (2000) 3242-3243. arXiv:http://dx.doi.org/10.1021/ja9939791, doi:10.1021/ja9939791.

[44] Z. Gan, Satellite transition magic-angle spinning nu- clear magnetic resonance spectroscopy of half-integer quadrupolar nuclei, J. Chem. Phys. $114 \quad$ (24) (2001) 10845-10853. arXiv:http://dx.doi.org/10.1063/1.1374958, doi:10.1063/1.1374958

[45] R. K. Harris, E. D. Becker, S. M. C. D. Menezes, P. Granger, R. E. Hoffman, K. W. Zilm, Further conventions for NMR shielding and chemical shifts IUPAC recommendations 2008, Solid State Nucl. Mag. 33 (3) (2008) 41 - 56. doi:https://doi.org/10.1016/j.ssnmr.2008.02.004.

[46] M. Bak, J. T. Rasmussen, N. C. Nielsen, SIMPSON: A general simulation program for solid-state NMR spectroscopy, J. Magn. Reson. 147 (2) (2000) 296 - 330. doi:http://dx.doi.org/10.1006/jmre.2000.2179. 